\title{
Restructurations, délocalisations : les nouvelles formes de l'action territoriale
}

\author{
Franck AGGERI, Frédérique PALLEZ *
}

\section{Introduction}

Les problèmes de restructuration et de délocalisation contribuent au fond permanent d'une inquiétude sociale, alimentée à intervalles réguliers par les media. Mais, au cours des dernières décennies, ils ont progressivement changé de nature, et parallèlement, les formes d'action publique ont elles aussi évolué.

Jusqu'aux années 70, les restructurations industrielles désignaient, en France, des phénomènes bien identifiés : elles concernaient un petit nombre de secteurs industriels dont l'adaptation paraissait douloureuse, mais inéluctable (textile, chantiers navals, sidérurgie); elles mettaient aux prises un petit nombre d'acteurs (entreprises, syndicats, Etat central); elles se déroulaient sous la houlette de l'Etat qui apportait à la fois les ressources essentielles et qui était porteur d'une vision en matière de politique industrielle et d'aménagement du territoire.

Aujourd'hui, le terme de restructurations industrielles, souvent remplacé par celui de mutations industrielles, évoque davantage une variété de phénomènes mal cernés dont on peine à dégager un modèle unique : un processus diffus et permanent d'adaptation des entreprises qui concerne tous les secteurs industriels ; la multiplication des parties prenantes et des groupes concernés par ces processus ; l'effacement progressif de l'Etat colbertien, au profit de formes plus a-centriques d'actions publiques.

\footnotetext{
* Centre de gestion scientifique de l'école des mines de Paris.
} 
La mondialisation, en particulier, a engendré une crise de l'action publique en matière de restructurations. En premier lieu, les politiques industrielles d'inspiration colbertiste - où l'Etat central, souvent actionnaire, tentait d'orienter l'action de l'entreprise - ont progressivement disparu dans une économie ouverte et dérégulée ; deuxièmement, la manne des préretraites s'est progressivement tarie du fait de leur coût prohibitif pour les finances publiques et les restrictions imposées par Bruxelles (78 000 préretraites aidées en 1998 contre 700000 en 1983) ; enfin, bien qu'en plein essor, la production de règles de droit, visant à encadrer les rapports sociaux et la négociation collective des plans sociaux, ne suffit pas face à la multiplication des débordements (troubles à l'ordre public, irruption du politique, etc.) et à l'intrication des registres (politique, économique, territorial, industriel, social, domestique, etc.). Ainsi, les situations de blocage et les conflits larvés se multiplient-ils sur le terrain, suscitant un nouvel appel aux pouvoirs publics.

Plus récemment, l'inquiétude liée aux processus de «désindustrialisation » supposés s'amplifier en Europe occidentale (Datar, 2004) a alimenté un retour de l'Etat sur le devant de la scène, autour de différentes initiatives, comme les pôles de compétitivité, la création de l'Agence de l'innovation industrielle, suggérant que sous des formes nouvelles, réapparaissait une sorte d'interventionnisme new look du niveau central.

Dans ce contexte, et au-delà des péripéties médiatiques, on constate que de nouvelles formes d'action publique, moins centralisées, mobilisant très largement les acteurs locaux (administrations déconcentrées, élus locaux, collectivités locales, organismes consulaires, etc.) s'inventent à bas bruit, depuis plusieurs années. Ce constat corrobore les observations d'autres chercheurs (Duran, Thoenig, 1996 ; Lascoumes, Le Bourhis, 1998) qui soulignent le développement de formes d'action publiques multipolaires et " procéduralisées ", se substituant au modèle où l'Etat central définissait de façon centralisée les objectifs et les moyens de l'action publique.

Mais le déploiement informel de ces initiatives, s'il permet de favoriser réactivité et souplesse, ne va pas sans poser de questions en termes d'équité (entre territoires, entre individus), de partage des responsabilités entre acteurs publics et privés, bref de principes guidant l'action collective. En outre, il pose le problème de la mutualisation et de la transposabilité de ces expériences qui, pour singulières qu'elles soient, cachent peut-être des idées à faire partager à tous.

Quelles sont les conditions d'émergence d'un apprentissage collectif qui permette de reformuler les représentations, les buts et les modalités d'une action collective, et qui évite de réinventer des solutions déjà expérimentées ailleurs? Tenter de répondre à cette question nous semble exiger, au 
préalable, une analyse précise des pratiques et des expériences innovantes qui s'inventent sur le terrain.

Dans ce texte, après avoir brièvement rappelé les éléments de contexte qui caractérisent à la fois les mutations industrielles et leur perception, nous traiterons deux exemples concrets issus d'études de terrain. Le premier concerne les actions de reconversion qui se sont développées sur le territoire choletais en réaction à la crise de la chaussure. Le deuxième concerne les actions visant à prévenir les délocalisations en Lorraine, dans la filière automobile. Dans une troisième partie, nous essaierons de caractériser de manière plus générale à la fois les traits essentiels et les conditions de développement de telles formes d'action collective.

\section{Les mutations industrielles et l'action publique : le contexte}

\section{I.1. les mutations industrielles : une idée admise, une lacune des instruments d'observation}

La restructuration est devenue un outil permanent d'adaptation industrielle des entreprises, à la recherche d'une compétitivité croissante, qui, en outre, est souvent pensée à une échelle transnationale, dans des centres de décision situés hors de nos frontières.

Le problème est qu'au-delà de ce constat général, généralement admis, la compréhension fine de ces phénomènes continus n'est possible qu'en spécifiant les logiques industrielles à l'œuvre, qui peuvent être relativement différentes selon les secteurs industriels concernés. Cette nécessaire complexité de l'analyse rend délicate l'appréhension des phénomènes, d'autant que leur perception est très partielle.

En effet, il est frappant de constater que la perception des phénomènes par l'opinion publique, modelée par les media et le discours des politiques, reste focalisée sur les évènements d'ampleur significative, notamment en termes d'emplois, qu'ils soient considérés comme positifs ou négatifs : les plans sociaux d'importance, les délocalisations ou les nouvelles implantations de grande taille. Or ces événements ne donnent pas la vision d'ensemble du phénomène, à plusieurs titres : ils ne sont que l'aboutissement de phénomènes continus plus invisibles ; ils masquent, d'autre part, les mouvements de reconfiguration plus modestes.

En effet, pour préciser le premier point, le plan social (plan de sauvegarde de l'emploi dans son libellé actuel) ne constitue qu'un moment du processus qui va de l'annonce d'un plan de restructuration par une entreprise jusqu'à sa mise en œuvre, même s'il est le moment le plus douloureux 
pour les salariés. En outre, les plans sociaux - qui représentent moins de $15 \%$ des licenciements pour motif économique - ne constituent que la face émergée de l'iceberg, la phase terminale de la restructuration, une fois que toutes les autres solutions visant à accroitre la flexibilité de l'emploi ont été épuisées : sous-traitance, intérim, CDD, cessations d'activité, etc.

Quant à la connaissance des opérations de restructuration plus modestes, leurs enjeux plus limités réduisent d'autant l'intérêt qui leur est porté sur le plan médiatique et politique, et, par conséquence, la précision, voire l'existence même d'instruments d'observation, en est affectée.

Symétriquement aux événements conduisant à des destructions d'emplois, l'attention se porte préférentiellement sur les opérations d'implantations nouvelles, significatives en termes de création d'emplois, alors que l'enjeu actuel est peut-être, au moins autant, le maintien d'emplois existants dans les entreprises déjà implantées, et donc l'ancrage de ces entreprises sur leur territoire. Le problème, on y reviendra, est que l'on ne peut facilement comptabiliser les emplois dont la destruction a été évitée.

\section{l-2. L'évolution des formes d'action publique}

Parallèlement à l'évolution des restructurations vers des mutations économiques, s'est opéré un retrait progressif de l'Etat, qui était en première ligne à divers titres : en tant qu'Etat-patron, dans un certain nombre de secteurs, mais aussi en tant que stratège industriel, et pourvoyeur de financements (notamment les pré-retraites), qui ont joué un rôle clé dans l'acceptabilité sociale d'un certain nombre de grandes opérations de restructurations des dernières décennies.

L'effacement de l'Etat colbertien au cours des vingt dernières années coïncide avec la montée en puissance de la régulation par le droit du travail qui vise à encadrer les rapports sociaux et la négociation collective. Cette régulation d'ordre juridique se poursuit depuis plus de vingt ans, sur le plan de la production législative mais également au travers de l'accumulation d'une jurisprudence importante.

Cette montée en puissance, jusques et y compris dans ses dernières manifestations (article L 118 de la loi de modernisation, lui-même transformé en L 321-17 en 2005) n'est pas exempte de critiques : son caractère procédurier, potentiellement inéquitable, limité en termes de registre d'action, contestable en termes d'efficacité, a souvent été mis en exergue.

Deux évolutions plus récentes sont toutefois à noter, qui viennent tempérer l'analyse qui vient d'être présentée, sans remettre en cause, pour l'instant, l'essentiel du cadre des pratiques en vigueur et des instruments disponibles : 
- d'une part, on assiste en matière de restructurations, à une évolution du cadre communautaire, qui, s'il comporte une part traditionnelle de directives et d'instruments financiers, homologues des formes d'action traditionnelles développées au niveau national, développe aussi des éléments au statut plus incertain, plus rhétorique que prescriptif (recommandations, consultations, agenda social et rapports divers), qu'on a pu qualifier de "soft law » (Triomphe, 2005).

- d'autre part, réémergent en France, mais aussi en Europe ${ }^{1}$, après une longue période de discrédit, des velléités de reconstituer une forme de politique industrielle, fondée sur un interventionnisme new look, appuyé sur l'incitation plus que sur la contrainte. Ses manifestations, encore embryonnaires, en sont variées : rapport Beffa et création de l'Agence de l'innovation industrielle, pôles de compétitivité, grands projets transnationaux.

Cela dit, quelles que soient ces évolutions, on observe que les cadres établis sont constamment débordés : d'une part, le recours à l'Etat, central ou déconcentré, constitue toujours une norme, lors de crises sociales liées à une restructuration importante... D'autre part, de nouvelles formes d'action collective territorialisées se développent, de manière plus subreptice, visant à recréer, à chaud, juste après une restructuration, ou plus à froid, en anticipation, une dynamique territoriale, et explorent des pistes peut-être prometteuses pour l'avenir. Mais leurs caractéristiques et leurs effets sont mal connus.

Il semble donc intéressant de repérer et de caractériser ces innovations locales, leur degré de généralité, et d'effectuer un retour d'expérience qui mette en évidence les marges de manœuvre dont peuvent disposer les différents acteurs locaux, dans des situations présentées comme très contraintes.

\section{Deux exemples d'action territoriale}

Nous nous appuierons sur deux cas, (issus d'études de terrain réalisées respectivement en 2000-2001 et en 2004), se situant à différents moments du processus de mutation évoqué en première partie. L'un concerne une crise issue de restructurations qui se sont succédées à un rythme rapide, dans le secteur de la chaussure, dans le Choletais. L'autre porte sur l'action menée en Lorraine, plus à froid, dans la filière automobile, face aux risques de délocalisation ressentis par les acteurs de terrain.

1. Une communication de la Commission, du 31/3/2005, met sur le même plan politique de la concurrence et politique industrielle. 


\section{II-1. La crise choletaise de la chaussure}

\section{Une crise majeure mais peu médiatisée}

Le Choletais est aux prises depuis la fin des années 90 à une crise massive de l'une de ses industries emblématiques : la chaussure. L'intensification de la concurrence des pays à bas coûts de main d'œuvre qui a accéléré le mouvement de délocalisation, la concentration de la distribution de la chaussure et des erreurs stratégiques de quelques entreprises phares, ont engendré la suppression, entre 1999 et 2001, de près du quart des effectifs du secteur (2 300 sur 9 500) et le dépôt de bilan de plusieurs entreprises. Malgré l’ampleur de la crise, sa médiatisation est restée faible. Le contexte économique prospère de la région (un des plus faibles taux de chômage en France), les licenciements diffus par petits flux, la faible conflictualité sociale, n'ont pas favorisé une mobilisation collective immédiate bien que ces mutations aient entraîné des drames locaux : des bourgs, vivant autour de leur usine de chaussure, ont été touchés de plein fouet, et les ouvrières de la chaussure, peu qualifiées et peu mobiles, ont eu de grandes difficultés à retrouver un emploi. Mais le Choletais a un atout par rapport à d'autres cas de restructurations diffuses : l'identification par tous les acteurs $-\mathrm{y}$ compris les pouvoirs publics - d'un territoire, doté d'une identité forte forgée au cours de l'histoire.

C'est donc par rapport à ce territoire qu'un certain nombre d'initiatives, aussi bien des collectivités territoriales que des services déconcentrés de l'Etat ou du niveau national, ont été lancées pour faire face à la crise. La panoplie des mesures de droit commun (pré-retraites, cellules de reclassement) est en effet apparue insuffisante pour faire face aux difficultés des salariés licenciés, et aux besoins de dynamisation économique du territoire. Mais les clivages politiques et les conflits d'intérêt s'opposaient au départ à la mise en cohérence de ces initiatives, dotées en outre de moyens assez limités. Ce n'est que progressivement qu'elles ont pris un sens global, à la faveur de l'émergence de dispositifs originaux reposant sur l'initiative de quelques individus qui se sont construits une légitimité et ont débordé du cadre classique de leur mission.

\section{Des dispositifs sur mesure}

Nous commenterons ici deux de ces dispositifs, la plate-forme de reconversion professionnelle, et le comité de pilotage de l'économie choletaise.

- La plate-forme de reconversion professionnelle est née du constat du caractère limité et peu coordonné de l'action des diverses cellules de reclassement, fonctionnant indépendamment sur des sites industriels pourtant situés sur un territoire restreint. Structure légère (1,5 personne au début), sa 
mission, relativement floue, et limitée au départ à la coordination et au relais en aval des cellules, s'est progressivement enrichie, sous l'impulsion d'un responsable au profil atypique, pour aller vers une véritable fonction d'intermédiation sur le marché du travail. Ainsi, pour reconvertir les ouvrières de la chaussure, il a fallu convaincre les entreprises déficitaires en main d'œuvre (mécanique, agro-alimentaire..) que des femmes pouvaient occuper des emplois destinés a priori à des hommes, qu'elles disposaient de savoir-faire importants, bien que peu visibles, et que moyennant des formations sur mesure elles seraient capables d'occuper les postes recherchés. D'autres blocages, comme la faible mobilité des salariés licenciés ou le financement des formations individuelles, ont du être surmontés par la mise en place de mesures spécifiques financées par le département et la région.

- Le comité de pilotage de l'économie choletaise est né de l'initiative du sous-préfet de Cholet, qui a su utiliser l'électrochoc de la crise pour mettre autour de la table les différentes parties prenantes (services déconcentrés, collectivités territoriales, organismes consulaires, etc.) et créer une communauté de travail informelle et multilatérale. La position de neutralité de la préfecture et la capacité de médiation personnelle du sous-préfet se sont avérées très utiles pour dépasser les conflits de personnes qui menaçaient la cohésion de ce comité. Progressivement, celui-ci s'est donné pour mission de produire un schéma stratégique de développement, et de réfléchir à la possibilité d'anticiper les reconversions des salariés dans les secteurs en mutation. Ainsi, petit à petit, le comité coordonne toutes les actions de développement sur le territoire (prospection de nouvelles entreprises, analyse prospective pour le développement économique et en matière d'infrastructures, etc.) qui émanent pourtant d'acteurs différents (comité d'expansion, CCI, région, département, communes, DATAR, etc.) et soucieux de leurs prérogatives. Ce comité rétablit donc la vision intégrée qui semble indispensable au traitement des mutations économiques, il tente de promouvoir une vision prospective qui dépasse la simple logique de réparation souvent mise en avant, il construit une structure susceptible de mener la « conversation avec la situation » (Schön, 1983) qu'implique la conduite d'un projet dans un univers incertain.

\section{Un bilan multi-facettes et inachevé}

En termes purement quantitatifs, et si l'on se limite au reclassement en CDI des salariés licenciés, les résultats obtenus grâce au Plan d'action pour l'économie choletaise peuvent paraitre modestes : $43 \%$ seulement des salariés qui sont passés par les cellules de reclassement ayant fonctionné en 2000 ont retrouvé un CDI en novembre 2001. C'est peu si on compare ce chiffre, par exemple, aux résultats obtenus lors du troisième plan social de l'entre- 
prise Chausson ( $75 \%$ des salariés licenciés ont retrouvé un emploi en CDI dans d'autres entreprises) (Bruggeman, 2001).

Cela dit, les actions entreprises peuvent également être jugées sous un autre angle : celui de l'enclenchement d'une dynamique territoriale. En effet, dans une situation qui, de toute façon, est loin d'être stabilisée puisque de nouveaux licenciements sont prévus, fonctionne maintenant une instance, le comité de pilotage, qui prend en charge collectivement les problèmes de développement économique, et qui, par une alchimie subtile, a acquis une légitimité incontestable : pour les élus du territoire, «il faut en être».

Dans le même esprit, la plate forme de reconversion professionnelle, structure en principe limitée dans le temps, pourrait voir prolonger son existence autour de missions davantage orientées vers la requalification à froid des salariés, c'est-à-dire avant un éventuel licenciement, explorant ainsi les voies de l'anticipation sous une forme pragmatique fort éloignée des incantations habituelles.

Nous sommes ainsi passés de l'évaluation de dispositifs de crise, à l'évaluation chemin faisant d'une action collective en train de se faire et de se définir.

Un problème essentiel demeure toutefois celui de la poursuite de ces formes d'action collective en routine, dans un contexte où les mutations industrielles se poursuivent sur le territoire, et où les solidarités qui avaient pu jouer à chaud ont plus de difficultés à se nouer dans l'action quotidienne.

Pour éclairer cette problématique, nous allons nous appuyer sur un autre exemple où, précisément, aucune crise ouverte n'est déclenchée, au-delà d'une inquiétude latente sur les délocalisations possibles.

\section{II-2. Les risques de désindustrialisation dans la filière automobile, en Lorraine}

\section{Les signaux faibles de la délocalisation?}

Il n'existe pas de preuves tangibles d'un processus de délocalisation massif dans l'industrie automobile en Lorraine, et plus généralement en France, comme l'ont connu le textile ou l'industrie de la chaussure. Toutefois on constate plusieurs évolutions défavorables : un ralentissement des nouvelles implantations en Europe de l'Ouest et leur essor en Europe de l'Est ainsi qu'un phénomène de délocalisation diffus qui se traduit par le transfert progressif d'activités au sein d'un même groupe vers des usines dans les pays low cost. Pourtant la Lorraine a été une terre d'accueil d'investissements automobiles dans les années 90 . 


\section{- Les (anciens ?) atouts du territoire}

Cette zone géographique, qui avait un tissu industriel automobile quasi inexistant au début des années 80 , est en effet devenue une des premières régions automobiles françaises avec 19000 emplois et plus de 70 entreprises en 2002. Il s'agit, pour l'essentiel, d'équipementiers appartenant à des capitaux étrangers, allemands en particulier, mais aussi américains ou canadiens ${ }^{1}$. Les facteurs les plus fréquemment cités pour justifier ces implantations étaient : une localisation géographique à proximité de nombreuses usines terminales des constructeurs, des coûts de main d'œuvre plus faibles qu'en Allemagne, des infrastructures de qualité, des aides publiques généreuses (primes d'aménagement au territoire), des terrains disponibles, le bilinguisme des populations, etc.

Pendant les années 90, un discours optimiste a prévalu parmi les pouvoirs publics et les experts selon lequel les risques de délocalisation étaient plus limités dans l'automobile que dans d'autres secteurs compte tenu de l'importance de deux facteurs : la proximité géographique par rapport aux usines terminales des constructeurs et les exigences en matière de qualité et de maitrise technologique qui seraient hors de portée des pays à bas coût de main d'œuvre. Les évolutions récentes de la filière automobile invitent à reconsidérer ce diagnostic et à étudier de manière plus fine et approfondie les stratégies de localisation des constructeurs et des équipementiers.

\section{- Des évolutions stratégiques défavorables au sein de la filière}

Les craintes de délocalisation sont alimentées par certaines évolutions dans la filière automobile comme la poursuite du mouvement d'externalisation, la nouvelle localisation des constructeurs automobiles qui se développent dans les pays émergents ou encore la rationalisation des équipementiers de rang 2 qui sont encouragés à se concentrer ${ }^{2}$. Mais, plus encore, ce sont les nouvelles doctrines des responsables achats, qui intensifient la pression sur les coûts auprès de leurs fournisseurs et qui se lancent dans une politique d'approvisionnement mondial (global sourcing), s'appuyant sur des antennes dans les pays low costs, qui inquiètent les acteurs publics territoriaux. Selon les achats, le seul véritable argument à long terme contre les délocalisations porte sur la logistique (coût et réactivité). Ainsi, plus les pièces sont volumineuses et fragiles, donc coûteuses à transporter, plus la diversité et les exigences de flexibilité sont fortes (livraisons en juste-à-temps, variations de

1. Parmi les filiales de groupes étrangers implantés dans cette zone géographique on peut citer MCC (Daimler-Chrysler) et Behr à Hambach, Continental (pneus) et Delphi (batteries) à Sarreguemines.

2. Pour une synthèse, voir Lung (2003). 
volume et de modèles), plus l'éloignement est un facteur de risque (rupture et coût logistique) pour le donneur d'ordre.

Il faut cependant relativiser la mise en oeuvre de ces doctrines d'achat. Ainsi, le mouvement de délocalisation est beaucoup plus lent que ne le souhaiteraient les directions des achats. Il faut notamment considérer les échecs répétés de certaines délocalisations d'équipementiers qui ne sont pas arrivés à remplir les cahiers des charges fixés par les donneurs d'ordre mais aussi les logiques contradictoires qui peuvent prévaloir au sein des donneurs d'ordre entre les achats qui privilégient la réduction des coûts d'une part, les services d'industrialisation ou les usines qui sont attentifs aux compétences, aux capacités de réactivité et à la proximité - y compris culturelle - des relations avec les sous-traitants. Sans compter les gains de productivité inattendus et les capacités d'innovation qu'ont su mettre en oeuvre, localement, certains équipementiers pour éviter la délocalisation.

\section{Des formes d'action collective à réinventer}

Comment échapper au déterminisme économique qui semble se mettre en oeuvre ? Face à ces évolutions défavorables, quels sont les leviers d'action des acteurs territoriaux ? Quels moyens peuvent-ils activer pour renforcer la compétitivité du territoire dans le secteur automobile ?

\section{- Des acteurs configurés pour attirer de nouveaux investissements}

Confrontée à la reconversion de ses anciennes industries (mines et sidérurgie), la Lorraine a bénéficié pendant vingt ans de conditions favorables pour attirer de nouveaux investissements : aux aides européennes (Feder), s'ajoutaient les aides directes et indirectes apportés par l'Etat, notamment primes à l'aménagement du territoire (PAT). En Moselle, où de nombreux équipementiers automobiles se sont installés, l'action du Fonds d'Industrialisation du Bassin de Moselle (FIBM) - une émanation de Charbonnages de France - a joué un rôle essentiel dans la structuration de l'action publique territoriale. Dans cette perspective, l'intervention publique portait sur trois points principaux : la prospection des investisseurs potentiels ; le montage de dossiers financiers; l'aménagement des terrains et le développement de nouvelles infrastructures.

\section{- La nouvelle problématique de l'ancrage territorial}

La situation est aujourd'hui beaucoup moins favorable. La fin des PAT et du FEDER objectif II en 2006 va réduire les marges de manœuvre financière. Le FIBM est appelé également à disparaître.

Par ailleurs, les acteurs territoriaux sont confrontés, lors de nouveaux projets d'investissement en France (nouveaux produits, projets d'extension), à un marchandage systématique de la part des industriels : arguant de rigidi- 
tés administratives et de coûts salariaux incompatibles avec les exigences de rentabilité de leurs actionnaires et les pressions de leurs clients, ils réclament des compensations (aides financières à la formation, négociation de la taxe professionnelle, infrastructures diverses). On pourrait ne voir dans ce marchandage généralisé que l'institutionnalisation des tentatives visant à obtenir des aménagements par rapport au cadre légal, mais la dégradation de la situation financière des fournisseurs de rang 2 en France indique que le phénomène est à prendre au sérieux.

Enfin, la plupart des aides étatiques ou européennes sont liées à un critère de création d'emplois. Les règles excluent ainsi certains projets, notamment les projets les plus capitalistiques. Ces aides sont également limitées en cas d'appartenance à un groupe, et ciblées sur certains types de dépenses. L'efficacité de l'action territoriale est également mesurée à l'aune du nombre d'emplois créés. Comme nous allons le voir, ce critère de jugement apparait de plus en plus inadapté avec la nature des dossiers traités et les marges de manoeuvre que ces acteurs peuvent mettre en oeuvre.

\section{- Des marges de manceuvre différentes selon le type de décision}

Les marges de manœuvre des acteurs territoriaux sont, en effet, différentes selon le type de décision industrielle qui est en cause :

- les marges de manoeuvre sont faibles dans le cas d'une nouvelle implantation. Les décisions sont guidées par les logiques d'achat et les stratégies des équipementiers et des constructeurs, au niveau des maisons mères, qui privilégient des grandes variables (coûts, zone géographique). Les acteurs locaux n'interviennent que pour départager des territoires comparables (la Lorraine n'est pas en concurrence avec la Chine mais avec d'autres régions françaises et d'Europe de l'Ouest).

- ces marges sont plus importantes dans le cas de projets d'extension ou pour éviter les projets de délocalisation. Ici, les acteurs territoriaux peuvent trouver un allié dans la personne du responsable du site industriel. Leur objectif commun est en effet de développer et pérenniser un site existant qui a déjà fait ses preuves. Ils peuvent aider les industriels locaux à vendre le territoire auprès de leur maison mère en valorisant les actifs intangibles : compétences, qualité du territoire... Plus concrètement, ils peuvent construire des soutiens sur-mesure qui manifestent symboliquement et financièrement la volonté du territoire de collaborer avec l'industriel (ex. financement d'adductions de fluides, de bâtiments ou d'infrastructures diverses, y compris équipements collectifs - crèches, salles polyvalentes -, aide à la formation du personnel,...), en faisant preuve de réactivité et d'adaptation aux besoins spécifiques des industriels. 
REVUE DE L'IRES N N $^{\circ}$ - 2005/1

\section{Quels leviers pour les acteurs territoriaux ?}

Avec la montée de la problématique de l'ancrage sur le territoire des entreprises, on assiste donc à une transformation du modèle d'action publique territoriale. On serait en train de passer d'un modèle où, face à de nouvelles implantations, génératrices d'emplois, se jouait l'attractivité d'un territoire, fondée essentiellement sur des aides financières, gérées selon des règles explicites et standardisées, à un modèle où l'enjeu principal est l'ancrage et le développement, non nécessairement créateurs d'emplois, d'entreprises existantes, sur la base de coopérations avec les acteurs locaux montées sur mesure par rapport aux besoins de chaque industriel. Or cette optique déstabilise les acteurs du développement territorial car elle remet en cause une partie de leurs modes de fonctionnement traditionnels. En outre, leur action devient plus difficile à visibiliser, à la fois vis-à-vis de leurs tutelles et vis-à-vis des élus locaux.

Les réponses de ces acteurs doivent donc maintenant être configurées selon des principes en apparence contradictoires : il leur faut prendre acte de la généralisation du marchandage, pour aller vers la personnalisation de l'offre faite aux industriels, tout en en limitant les effets pervers, notamment en termes de surenchère entre territoires proches, d'incohérence entre les acteurs publics et d'inéquité flagrante entre industriels sur un même territoire.

La construction d'une offre personnalisée lors de nouveaux projets d'investissement (renouvellement, productivité, extension, développement,..) passe par l'identification préalable, en continu, des besoins ou des points faibles (main d'oeuvre, compétences, formation, offre foncière et immobilière, aides financières, infrastructures,..), sur lesquels construire des propositions. A l'aide de cette connaissance, peut ensuite se déployer une certaine inventivité dans la mise en place d'une ingénierie sur mesure. L'examen de l'action publique territoriale sur différents projets d'investissement nous a conduits à identifier quatre volets sur lesquels cette ingénierie existe déjà, et pourrait être renforcée :

\section{- L'ingénierie financière}

L'ingénierie financière, structurée au fil du temps, a reposé sur l'existence, pendant longtemps, d'aides nationales et européennes, mais tient également à des acteurs - sociétés de conversion - qui ont développé des compétences spécifiques à ce sujet. La restriction des aides, ou le retrait de ces acteurs pourrait à cet égard priver les territoires d'un atout fondamental car, outre l'effet direct du levier financier, cette ingénierie est un outil puissant de connaissance des situations industrielles locales. 


\section{- L’ingénierie foncière et immobilière}

L'ingénierie foncière et immobilière s'est développée au sein des collectivités territoriales, et plus spécifiquement des intercommunalités, sur la base de création de zones industrielles équipées. La constitution d'outils comme les sociétés d'économie mixte, outils jugés plus souples pour gérer l'offre foncière, permettant de prendre en charge la construction de bâtiments et de les proposer ensuite en crédit-bail aux entreprises, semble répondre à une tendance actuelle des entreprises, qui tentent, dans tous les domaines, d'externaliser les tâches qui ne sont pas de leur cœur de métier. On observe également de plus en plus fréquemment que l'offre foncière et immobilière, dans les dossiers actuels, est elle-même maintenant conçue comme un package, incluant, au-delà du terrain et du bâtiment industriel correspondants au cahier des charges, différents à-côtés négociés au coup par coup. Ces éléments, qui font objectivement baisser le coût de l'opération foncière et immobilière pour l'industriel, ne donnent pas lieu à consolidation, et permettent ainsi de personnaliser l'offre faite aux entreprises.

\section{- L’ingénierie des compétences}

Avec la disparition prévisible des aides financières classiques, c'est probablement, aux yeux des industriels, un domaine sur lequel, en contrepartie, ils attendent prioritairement un soutien territorial. Les choix d'implantation, et les décisions d'investissement sur un site existant, s'appuient en effet sur la possibilité de recruter, de retenir et de faire progresser en compétence les salariés de l'entreprise. Ce souci est très présent chez les industriels au moment des implantations, et peut les conduire à renoncer à un site où ils considèreraient que le bassin de main d'œuvre disponible est insuffisant ou mal formé. En outre, la formation est un levier qui, pour l'instant, ne tombe pas sous le coup de réglementations européennes, étant considérée comme une aide individuelle, et non une aide collective à l'entreprise. Plus généralement, les entreprises semblent attendre non seulement une aide financière sur ces questions, mais une véritable compétence d'ingénierie et d'ensemblier, d'autant plus sensible que les acteurs de l'enseignement et de la formation sont assez dispersés, et que les besoins sont spécifiques.

\section{- Une organisation collective de la réactivité}

Le dernier volet porte sur l'organisation collective de la réactivité. Un nouveau projet d'investissement requiert, en effet, une multitude d'autorisations administratives dont la complexité rebute l'industriel mais dont, en outre, les délais sont généralement difficilement compatibles avec son horizon de gestion. La capacité de mobilisation collective des acteurs territoriaux pour gérer ces dossiers et en accélérer la mise en oeuvre semble avoir joué, à cet égard, un rôle non négligeable dans la conclusion de nouveaux projets. 


\section{Une coordination à pérenniser dans le temps}

Différents exemples concrets montrent que ces leviers ont déjà été mobilisés, parfois avec succès, pour favoriser des projets d'extension ou éviter des projets de délocalisation où les industriels locaux étaient en concurrence avec d'autres sites appartenant au groupe. C'est toutefois de la coordination et de la cohérence de ces actions territoriales que pourra résulter une plus grande efficacité de leur action, dans un contexte où, les ressources devenant plus rares, l'optimisation de leur utilisation devient critique.

\section{Les nouvelles formes d'action collective territoriale}

Au-delà des différences apparentes de ces deux cas, on repère un certain nombre de traits qui leur sont communs, et qui pourraient permettre d'ébaucher une forme, peut-être généralisable, de ces actions qui se développent au-delà des dispositifs juridiques et économiques classiques. Une des questions essentielles est en effet, à supposer que l'on ait démontré l'intérêt et l'efficacité de ces initiatives, d'en étudier les conditions d'émergence et les traits principaux, pour en envisager la reproductibilité.

\section{III.1. Les conditions d'émergence d'une action collective territoriale}

Les formes d'action telles que celles que nous avons décrites précédemment n'ont rien de naturel. Elles ne naissent pas partout et, sur beaucoup de territoires, les acteurs subissent, isolément, les conséquences des mutations industrielles, sans que s'organisent collectivement les réponses aux crises territoriales.

Une explication de cet état de fait réside dans la constatation, classique, de l'existence de puissantes logiques institutionnelles qui ne poussent pas les acteurs à la coopération et qui les font agir, chacun dans son domaine de compétence, et avec les instruments dont il dispose. Or, on l'a vu, la réponse aux problèmes provoqués par les mutations industrielles est transversale et multi-facettes, elle touche autant à des problèmes d'emploi que d'économie, elle traverse les frontières administratives des territoires et des services de l'Etat. Bien sûr, cette description peut sembler caricaturale car se mettent en place des dispositifs d'organisation de la coopération ou, au minimum, d'information réciproque. Elle n'en est pas moins un facteur d'explication de nombreuses situations constatées localement.

\section{Des solidarités historiques}

Ces logiques ont d'ailleurs joué dans les deux cas évoqués précédemment. Mais elles ont pu être dépassées à certains moments. Pourquoi ? Dans les deux cas, nous semblent jouer, d'abord, des solidarités historiques qui 
sont très spécifiques aux territoires concernés, solidarités latentes ${ }^{1}$ qui, anciennes, renaissent à l'occasion de l'électrochoc de la crise, dans le Choletais, ou qui, liées à une histoire politique et industrielle plus récente, comme en Moselle, se mobilisent, sous l'impulsion d'un acteur industriel prépondérant comme Charbonnages de France.

\section{La permanence des acteurs}

Mais plus que cette mobilisation, c'est sa durée qui pose problème. Dans le Choletais, une fois la situation de crise rentrée dans une sorte de permanence, on a pu constater que le problème était de continuer à mobiliser des acteurs qui ne joueront pas nécessairement éternellement le jeu de la coopération. En Moselle, la présence et l'action continue de Charbonnages de France, depuis des décennies, a permis la mise en place de dispositifs d'intervention (via, notamment, sa filiale, société de conversion) qui lui donnent un rôle fédérateur officieux, et surtout un droit de regard sur les situations et les politiques industrielles, source de connaissances précieuses pour l'action territoriale.

Dans les deux cas, on pressent que la continuité des acteurs, non pas seulement en tant qu'institutions, mais surtout en tant qu'individus, est une condition indispensable car les initiatives que nous avons décrites reposent sur l'organisation et l'animation d'un réseau, qui rend possible le développement d'une compétence collective. Or le bon fonctionnement de ce réseau est lié aux relations interpersonnelles et à l'engagement des individus, qui doivent pouvoir être identifiés et stabilisés un minimum. Cette continuité n'est pas nécessairement acquise, quand elle se heurte à certaines pratiques des gestion des personnes, notamment dans les services déconcentrés de l'Etat, où le turn-over des hauts fonctionnaires est délibérément important. Cela dit, continuité n'implique pas ossification, et il importe de veiller aussi aux passages de relais entre générations, pour éviter de voir disparaitre brutalement une compétence collective lors de départs en retraite.

\section{L'animation du réseau}

Mais l'animation du réseau ne résulte pas mécaniquement de la permanence des individus, elle doit aussi s'organiser en construisant des occasions concrètes de rencontre et d'échange qui soient utiles aux différentes parties prenantes, et équilibrées en termes d'échange d'information. C'est pourquoi les instances et les lieux neutres comme les préfectures, ou les agences de développement territorial, émanation des collectivités territoriales, sont parti-

1. Qui s'expriment sous une autre forme, dans la vie associative, par exemple. 
culièrement adaptés pour organiser cette animation, qui semble d'autant plus efficace qu'elle ne repose pas sur des dispositifs trop formalisés.

\section{Les responsables industriels locaux, des alliés à mobiliser}

L'efficacité de l'action de ces réseaux est en outre amplifiée par la mobilisation d'autres types d'acteurs, à savoir les industriels du territoire. On observe en effet, aussi bien dans le Choletais qu'en Lorraine, que des capacités de mobilisation a priori insoupçonnées existent chez des responsables de sites locaux, qui sont prêts à travailler à l'ancrage de ces sites pour s'opposer aux choix plus lointains et plus désincarnés des maisons mères. Ces responsables deviennent alors des alliés précieux pour le développement du territoire, car ils peuvent mobiliser des informations et des raisonnements inaccessibles aux acteurs publics.

\section{L'attracteur financier}

Enfin, il ne faut pas sous-estimer l'effet de ce que nous appellerons l'attracteur financier. Puissant moyen de fédérer des énergies, support incontournable des négociations avec les entreprises, mais également moyen d'organiser la vigilance sur leur situation grâce à la position de créancier, c'est un levier probablement indispensable pour faire émerger les initiatives locales, et ensuite, maintenir la compétence collective acquise à travers ces initiatives. De ce point de vue, l'encadrement de plus en plus strict des aides par les règles européennes, le retrait progressif de certains grands acteurs traditionnels (comme Charbonnages de France) pourrait être néfaste à la dynamique recherchée.

\section{III.2. Des actions collectives procédurales}

Après avoir indiqué les principales conditions d'émergence de ces formes d'action, comment les caractériser ? Nous retiendrons quelques points communs qui nous semblent dessiner un nouveau modèle d'une action collective procédurale.

\section{Vigilance, plus qu'anticipation?}

L'anticipation est souvent entendue comme la prévision du moment où un industriel annoncera une opération de restructuration, l'idée communément admise étant que cette information permettra de passer d'une gestion de crise à une stratégie plus sereinement conçue et conduite. Or, comme nous avons déjà eu l'occasion de l'expliquer (Aggeri, Pallez, 2003), un industriel n'a jamais intérêt, sauf s'il y est obligé juridiquement ( $f f$. le cas de la Suède) à effectuer ce type d'annonce prématurément, car elle accélèrera et amplifiera ses difficultés. Par ailleurs, différents exemples montrent que la 
préparation anticipée des esprits ne le met pas à l'abri de réactions violentes au moment des décisions finales.

Mais, plus fondamentalement, il nous semble qu'il y a derrière cette notion d'anticipation une vision presque météorologique des phénomènes, au sens où l'on ne pourrait que les prévoir sans pouvoir agir sur eux. Or le cas lorrain nous montre qu'à l'inverse, c'est en organisant la vigilance sur l'évolution des situations des industriels du territoire qu'on peut construire avec eux des stratégies d'ancrage qui pourront prévenir, dans certains cas, les restructurations douloureuses.

L'organisation de cette vigilance est fondée à la fois sur une connaissance approfondie des évolutions spécifiques des filières représentées sur le territoire, mais aussi sur des contacts interpersonnels suffisamment fréquents avec les industriels, pour jauger leurs points faibles, leur proposer des moyens de les surmonter, et les aider à construire les argumentaires efficaces vis-à-vis de leurs sociétés mères.

\section{Le dépassement de la frontière privé-public, expression d'un principe de responsabilité partagée}

Les actions que nous avons décrites mettent en jeu des acteurs variés, services déconcentrés de l'Etat, collectivités territoriales, organismes de développement territorial, sociétés de conversion, entreprises, etc. Non seulement elles n'émanent pas que de l'Etat, mais elles se déploient sans souci de la traditionnelle frontière entre acteurs publics et privés, qui disparait, d'autant plus que, comme on l'a vu, l'action pour être pertinente nécessite d'être construite en collaboration étroite avec les industriels locaux. Cette prise de conscience a aussi été le fait de grands groupes industriels, publics ou privés, qui se sont impliqués dans le développement local par l'intermédiaire de filiales créées à cet effet (Raveyre, 2001). La responsabilité et la légitimité des entreprises sur ces problématiques est ainsi réaffirmée, mais le rôle des acteurs publics ou assimilés est essentiel pour stimuler cette responsabilisation, et rôder les coordinations nécessaires pour inventer les réponses pertinentes aux yeux des industriels.

\section{La construction de la coopération : un processus politique et interpersonnel}

La construction d'une compétence collective et d'une coopération indispensables pour apporter des réponses à des problèmes qui débordent des cadres traditionnels passe, on l'a dit, par l'organisation et l'animation d'un réseau entre les différents acteurs évoqués plus haut. Ce processus est à la fois un processus politique et interpersonnel. 
Cela dit, il nous semble que les modes de fonctionnement en sont fondamentalement différents selon que l'on gère une crise, à chaud, ou que l'action se développe à froid. Dans le premier cas, l'exigence de réactivité et de visibilité de l'action nécessite sans doute que le réseau se constitue plutôt en task force, animée par un chef de projet. Différents exemples, dont le cas choletais, montrent que ce chef de projet n'est pas désigné par son appartenance institutionnelle et ne peut qu'émerger, soutenu par une légitimité et un engagement personnels. Mais les deux cas étudiés nous suggèrent également que cette configuration de l'action doit sans doute laisser la place à un fonctionnement en réseau plus lâche, quand on n'est pas (ou plus) directement en situation de crise. Il est de fait que ce mode de fonctionnement est plus délicat à pérenniser et à justifier, compte tenu des risques de cloisonnement et d'inefficacité. Mais la nécessité d'un chef de projet n'est plus aussi flagrante. Par ailleurs, (en allant ainsi à l'encontre de l'idée de guichet unique) il nous semble que la multiplicité des points d'entrée dans le réseau devient alors un atout pour capter l'information et explorer les problèmes spécifiques de chaque industriel.

\section{De nouvelles ingénieries à susciter, des dispositifs organisationnels sur mesure}

La nature des problématiques mises en évidence, le caractère de moins en moins standardisé des demandes et des besoins détectés chez les interlocuteurs industriels, et les négociations très personnalisées qui se mènent avec eux, militent pour la constitution ou le renforcement de nouvelles ingénieries, appuyées sur les compétences collectives déjà mentionnées, et sur des dispositifs organisationnels ad hoc.

Par exemple, en matière d'ingénierie des compétences, on a constaté, sur le cas lorrain, combien la détection de besoins de formation, liés à un processus d'automatisation, et la construction d'une réponse à ces besoins, pouvait être un élément d'ancrage des entreprises sur un territoire. De même, dans le Choletais, le travail de la plate forme de reconversion, consistant à rapprocher les compétences et habiletés détenues par les ouvrières de la chaussure, et les besoins en main d'œuvre d'industriels d'autres secteurs (mécanique), pour permettre la reconversion des premières, est une autre illustration de la nature et de l'intérêt de cette ingénierie.

Il s'agit dans les deux cas, de mettre en relation des demandes de qualification exprimées par des industriels avec des compétences existantes, ou à développer, puis de proposer le montage, à la fois organisationnel et financier, qui permette ce rapprochement. Ce type d'action, ne peut se développer qu'en s'appuyant sur des dispositifs organisationnels qui en permettent la réalisation concrète, mais qui se révèlent être avant tout des supports à 
l'action d'individus, et qui à ce titre, sont, comme, comme on l'a montré pour la plate forme du Choletais, moulés en quelque sorte sur la personne qui les porte, construits sur mesure pour soutenir son action. D'où la déception que l'on peut ressentir à dupliquer ce type de dispositifs, qui ne constituent en rien une boîte à outils prête à l'emploi, mais qui doivent être réinventés dans chaque contexte.

\section{Une évaluation à repenser en cohérence avec les formes actuelles des mutations}

Enfin, une pierre angulaire de ces nouvelles formes d'action réside dans leurs modes d'évaluation. Ces modes d'évaluation, qu'ils concernent des actions ponctuelles, notamment après crise, ou l'action en routine de telle ou telle organisation, ont pour effet général de structurer le regard porté sur l'activité, et donc d'orienter les comportements. Or un des critères dominants d'évaluation de l'action dans ces domaines est, on l'a mentionné, le nombre d'emplois (créés, perdus, ...). C'est notamment pour cette raison que les opérations ayant un fort impact médiatique sont les restructurations massives ou les grosses implantations.

Or, dans les configurations étudiées, ce critère est particulièrement peu adapté pour au moins trois raisons :

- outre le fait que la qualité des emplois ne rentre pas en ligne de compte dans ce type d'évaluation, la comptabilisation brute des emplois créés, lors d'opérations de reclassement, conduit à des chiffres modestes difficiles à valoriser, en regard des efforts qui y ont été affectés ;

- quant aux actions ayant pour but l'ancrage des entreprises, donc le maintien d'un certain nombre d'emplois sur le territoire, leur évaluation est extrêmement difficile en raison des multiples facteurs jouant sur la situation, de la difficulté de déterminer une situation de référence, de l'impossibilité d'appréhender des relations causales simples entre les phénomènes. C'est la raison pour laquelle des controverses peuvent se développer sur l'évaluation d'une action ${ }^{1}$.

- en outre, dans un fonctionnement collectif, il devient par nature impossible d'attribuer à tel ou tel acteur institutionnel les résultats éventuellement constatés. Or ces acteurs, qu'ils soient publics ou privés, ont à justifier leur action devant leur tutelle ou leur hiérarchie.

Ces difficultés de l'évaluation et ses effets éventuellement contre productifs conduisent à souligner la nécessité d'une évaluation qualitative, complémentaire de l'évaluation quantitative qui de toute façon ne peut disparaitre compte tenu des fonctionnements organisationnels. Cette évaluation

1. Nous rejoignons là le point de vue souvent développé par Ginsbourger (2001). 
qualitative collective, sous forme de récits ou d'enquêtes, est en outre un moyen de mutualisation des expériences et de formation des acteurs. A ce double titre, elle semble donc une condition essentielle de développement de ces nouvelles formes d'action collective.

\section{Conclusion : de nouveaux objets de gouvernement}

Une caractéristique essentielle des actions que nous avons analysées réside dans le déplacement de l'action sur de nouveaux objets de gouvernement, déplacement visible dès que l'on abandonne une perspective limitée à une gestion de crise au profit d'une vision portant sur un horizon temporel élargi.

En fait, articuler logiques de court et de long terme requiert de dépasser la représentation traditionnelle de l'action publique qui consiste à raisonner sur deux objets distincts : l'emploi et l'entreprise. D'un côté, il est question de sauver les emplois, de reclasser les personnes, de l'autre, d'aider ou de créer des entreprises. Cette séparation, très ancrée dans les représentations, est renforcée par les découpages administratifs et juridiques. Si ces objectifs sont légitimes à chaud, les catégories que ces discours manipulent ne semblent plus pertinentes pour l'action de long terme : c'est la trajectoire de l'individu, comportant une succession d'emplois, mais aussi, le cas échéant, de formations ou d'inactivité, de transitions professionnelles, qui devient signifiante dans un monde plus mobile ; c'est, parallèlement, l'évolution économique d'un territoire que l'action collective peut infléchir, plus que la vie ou la survie d'entreprises dont la pérennité ne peut plus être considérée comme une donnée ou une fin en soi. Du point de vue de l'action collective, ces deux objets sont en outre indissociables : les trajectoires individuelles prennent sens par rapport aux spécificités du territoire, tant sur le plan des qualifications recherchées par ses entreprises que sur celui de l'offre de formation disponible ; réciproquement, les trajectoires économiques d'un territoire se construisent notamment à partir des compétences existantes ou émergentes.

Il nous semble donc que le lien entre l'action à court terme et l'action continue peut être renoué si l'action collective se donne explicitement comme but de raisonner sur le couplage entre ces deux « objets de gouvernement ${ }^{1}$ que sont les trajectoires individuelles et l'évolution économique des territoires. La crise n'est plus alors qu'une séquence de transition entre

1. Ce concept introduit par Foucault (1994) a été repris dans une perspective gestionnaire par Lenay (2001). 
deux moments d'une même trajectoire qu'il s'agit de mieux piloter globalement.

Une telle manière de voir a des conséquences concrètes : ainsi, si le développement territorial devient un objet plus pertinent que l'entreprise, il en résulte que, par exemple, l'aide apportée aux entreprises doit peut-être devenir plus sélective, et que les politiques d'ancrage mises en œuvre au niveau local se révèleront d'autant plus efficaces qu'elles seront hiérarchisées et différenciées selon les cas. A cet égard, aussi bien dans le cas choletais que dans le cas lorrain, il apparaissait assez vite que certaines stratégies industrielles pouvaient être soutenues avec un espoir de succès, alors que d'autres étaient vouées à l'échec.

De même, en matière de gestion des compétences, l'enjeu devient, non de tenter de conserver les emplois en soi, mais de solidifier, développer et visibiliser les compétences qui seront à la fois utiles aux industriels et réemployables ailleurs.

La question cruciale qui se pose alors est de savoir, au-delà d'expériences fragiles et isolées, fruit de circonstances particulières, comment consolider les cadres d'une action collective sur ces nouveaux objets, plus pertinents sur la durée, plus cohérents avec le fonctionnement des logiques industrielles et de leurs conséquences en termes de mutations, alors même que la mobilisation des acteurs, à son comble pendant la crise, se focalise en général essentiellement sur les enjeux à court terme, exprimés dans les anciennes catégories que sont l'entreprise et l'emploi. Une des réponses à cette question réside sans doute dans la conception de procédures d'évaluation qui soient plus en adéquation avec ces évolutions. 


\section{Références bibliographiques :}

Aggeri F., Pallez F. (2003), « Restructurations industrielles et mondialisation : une reconversion aussi pour l'Etat ? ", Politiques et management public, $21\left(n^{\circ} 2\right)$

Bruggeman F. (2001), « Analyse de l'exécution d'un plan : l'exemple de l'entreprise Chausson à Creil », in Le salarié, l'entreprise, le juge et l'emploi, Kerbourc'h J.Y. et Willmann C., Paris, La Documentation française.

DATAR (2004), « La France, puissance industrielle », La Documentation française, mars.

Duran P., Thoenig J.C. (1996), « L'Etat et la gestion publique territoriale », Revue française de sciences politiques, $n^{\circ} 4$, août, pp.580-623.

Foucault M. (1994), La gouvernementalité. Dits et écrits, vol. 3, pp.635-658, Gallimard.

Ginsbourger F. (2001), Traducteurs, traduction, Rapport au secrétariat d'Etat à I'Industrie.

Lascoumes P., Le Bourhis J.P. (1998), « Le bien commun comme construit territorial. Identités d'action et procédures », Politix, n42, pp 37- 66.

Lenay O. (2001), Régulation, planification et organisation du système hospitalier. La place des outils de gestion dans la conception des politiques publiques, Thèse de sciences de gestion, Ecole des Mines de Paris.

Lung Y. (2003), « The Challenges of the European Automotive Industry at the Beginning of the 21st Century ", Actes du GERPISA, n³5, décembre.

Raveyre M. (2001), « Implication territoriale des groupes et gestion du travail et de l'emploi. Vers des intermédiaires en réseau », Revue de l'IRES, $n^{\circ} 35$.

Schön D.A. (1983), The Reflective Practionner : How Professionals Think in Action, New York, Basic Books.

Triomphe C.E. (2005), Restructurations et cadres d'intervention publique en Europe, Communication au séminaire du 14 avril IRES. 